\title{
Juifs du Maghreb : onomastique et langue, une composante berbère?
}

\section{J. Taïeb}

\section{OpenEdition}

1 Journals

Édition électronique

URL : http://journals.openedition.org/encyclopedieberbere/1373

DOI : 10.4000/encyclopedieberbere.1373

ISSN : 2262-7197

Éditeur

Peeters Publishers

\section{Édition imprimée}

Date de publication : 1 mai 2004

Pagination : 3969-3975

ISBN : 2-7449-0452-X

ISSN : 1015-7344

\section{Référence électronique}

J. Taïeb, « Juifs du Maghreb : onomastique et langue, une composante berbère ? », Encyclopédie berbère [En ligne], 26 | 2004, document J17, mis en ligne le 01 juin 2011, consulté le 14 décembre 2020. URL : http://journals.openedition.org/encyclopedieberbere/1373 ; DOI : https://doi.org/10.4000/ encyclopedieberbere.1373

Ce document a été généré automatiquement le 14 décembre 2020.

(c) Tous droits réservés 


\title{
Juifs du Maghreb : onomastique et langue, une composante berbère?
}

\author{
J. Taïeb
}

Une composante berbère à l'origine des Juifs du Maghreb, parmi d'autres, comme l'évidente ascendance hébraïque? Cette assertion, maintes fois assénée par de nombreux historiens, faisait, il y a peu, figure d'article de foi. Des travaux, relativement récents, la remettent cependant en cause.

2 Basée, presque uniquement, sur les travaux de l'historien arabe Ibn Khaldoun (1332-1406), cette affirmation, précise, et étayée par de multiples détails, reprenait, en les rationalisant, des traditions orales immédiatement postérieures aux débuts de la conquête arabe du Maghreb (extrême fin du vII siècle, début du viII ${ }^{e}$ siècle). Ibn Khaldoun semblait faire sienne l'idée selon laquelle la Kahéna, la reine des Aurès, à la tête de la tribu des berbères Jarâwa, aurait pratiqué le judaïsme ainsi que ceux de sa tribu, la conversion s'étant effectué à une époque fort antérieure à la conquête arabe. D'autres groupes berbères auraient suivi la même évolution comme les Bahlula des confins algéro-marocains, les Madyûna de la région de Tlemcen, les Nefûsa des montagnes au sud de Tripoli.

Opposant une résistance acharnée aux conquérants arabes, la Kahéna, à la veille de sa mort et de sa défaite, aurait, pressentant son destin, adjuré ses fils de passer à la nouvelle foi et, graduellement, les Berbères, peu ou prou judaïsés, se seraient faits musulmans dans les premières décennies du viII ${ }^{\mathrm{e}}$ siècle. Cette thèse a été contestée par l'historien israélien Haïm Zeev Hirschberg (1974) à cause de la fragilité de l'information (le seul Ibn Khaldoun écrivant de longs siècles après les événements), du silence total des sources juives médiévales, en particulier celles de la Gueniza du Caire, de l'absence du moindre texte en berbère dans les documents juifs du haut et du bas Moyen Âge alors qu'abondent ceux écrits en arabe.

4 Mieux encore, l'affirmation d'Ibn Khaldoun, ou supposée telle, reposait sur une erreur de traduction. Ce dernier en effet déclarait simplement que certains Berbères avaient été, peut-être, (rubbama) judaïsés dans un lointain passé mais qu'ils n'étaient plus juifs à la veille de la conquête arabe (M. Talbi, 1971). 
5 Il n'est cependant pas impossible que des Berbères se soient convertis au judaïsme avant cette conquête mais c'est plus modestement une hypothèse, certes étayée sur de solides présomptions, mais sans preuves certaines. Parmi ces présomptions figure, en bonne place, l'abondance, encore que relative, des patronymes berbères portés par les Juifs du Maghreb et le fait, récemment mis en valeur, que certaines populations juives du Maghreb contemporain avaient le berbère comme langue de contact avec le milieu musulman environnant berbé-rophone, voire, pour une partie d'entre elles, comme langue usuelle à l'intérieur de leur communauté et langue de culture. Il faut enfin faire une place à un simple argument de bon sens, retenu par Haïm Zeev Hirschberg luimême, à savoir qu'Ibn Khaldoun et ses devanciers n'avaient pu tout inventer ( $\mathrm{H}$. Hirschberg, 1974, p. 145).

6 Sans entrer directement dans la querelle, nous chercherons simplement à déterminer d'abord la place chiffrée occupée par les patronymes berbères dans l'onomastique judéo-maghrébine. On tentera ensuite de préciser les zones géographiques où existaient, jusque vers 1950, des populations juives berbérophones. On ciblera en troisième lieu les terres d'élection de ces patronymes à l'intérieur de l'espace maghrébin. On hasardera, dans un quatrième temps, quelques hypothèses sur les déplacements de population. On démêlera enfin, avec prudence, les significations éventuelles des noms de famille.

\section{Les données statistiques}

7 En 1936, le grand rabbin Maurice Eisenbeth dénombrait 1146 souches pour trois pays du Maghreb: Tunisie, Algérie, Maroc et 4063 patronymes. Une souche peut en effet comporter plusieurs patronymes. Par exemple, le nom Hayoun ou Ayoun, avec l'indice de filiation arabe ben, devient Benayoun et, avec l'indice de filiation berbère ou, Ohayoun (ou-hayoun). On peut leur ajouter Layani ou Lahyani qui provient de la même racine (l'idée de vie en arabe).

8 La recension de $\mathrm{M}$. Eisenbeth était cependant incomplète car manquaient certaines souches, en Tunisie comme au Maroc, sans parler de la Libye, totalement absente. D'autres publications, depuis lors, ont permis de combler ces manques, combinées à quelques recherches personnelles (A. Laredo, 1978, J. Toledano, 1983).

9 En écartant de nos calculs un certain nombre de souches d'apparition récente, c'est-àdire contemporaines de la colonisation, on peut penser qu'il existait, pour l'ensemble des quatre pays du Maghreb (Libye, Tunisie, Algérie, Maroc), quelque 1400 souches dans les premières décennies $d u x_{x} x^{e}$ siècle, ce chiffre étant à prendre comme un simple ordre de grandeur.

10 Sur 1400 souches, nous avions, il y a peu, dénombré 83 qui étaient d'origine berbère et 64 qui avaient soit une origine berbère partielle par exemple Ohayoun, terme composite berbéro-arabe, soit une possibilité voire une certaine probabilité d'être d'origine berbère (J. Taïeb, 1998). Depuis lors, des compléments d'enquête auprès de nouvelles publications (J. Toledano, 1998) nous ont permis d'arriver à un total de 82 souches pour le premier groupe et de 68 pour le second.

11 On aurait donc approximativement une proportion voisine de $6 \%$ de souches certainement berbères et le pourcentage avoisinerait $11 \%$ si l'on tenait compte des souches partiellement berbères et d'autres vraisemblablement berbères. Ces chiffres, 
nous en convenons, ne sont en rien hégémoniques, ils sont loin cependant d'être insignifiants. Surtout, de nombreux patronymes, classés comme berbères, étaient très fréquents, comme Azoulay, répandu dans tout le Maghreb ; Sfedj ou Sfez, Guedj ou Guez très communs dans l'est de l'Algérie et en Tunisie; Amsalem, Amsallag, Azencot, Benguigui, Tedghi abondants au Maroc et dans l'Ouest algérien et nous en oublions.

Cette proportion de $11 \%$ est cependant d'un poids très inférieur à celle des souches arabes - quelque 37 ou $38 \%$ de manière certaine 45 à $50 \%$ en intégrant des souches partiellement arabes ou vraisemblablement arabes. Comme, d'autre part, les patronymes arabes sont extrêmement portés, leur fréquence au sein de la population dépasse probablement les $60 \%$. Dans des villes comme Alger, Tripoli, et surtout Tunis, cette proportion est même dépassée. Dans la dernière ville, si l'on fait abstraction des Juifs venus de Livourne à partir du XVII ${ }^{\mathrm{e}}$ siècle, il apparait que les noms de famille les plus fréquents, à quelques exceptions près, se réfèrent à l'arabe (J. Taïeb, 1997).

Il s'agit ici essentiellement des patronymes des vieux citadins, riches ou pauvres, enracinés dans la grande ville avant le xvIII ${ }^{\mathrm{e}}$ siècle, voire avant le $\mathrm{xvII}^{\mathrm{e}}$ siècle. À côté de noms comme Ghez ou Guez, Sfez probablement berbères, Sarfati d'origine hébraïque, Journo d'origine italienne, les autres sont, dans leur grande majorité, tirés de souches arabes comme Abitbol, Attal, Bellaïche, Bessis, Bismuth, Brami, ou Bramli, Chemla, Dana, Ganem ou Ghanem, Haggege, Hayat, Krief, Maarek, Naccache, Nataf, Nizard, Saada, Samama ou Scemama, Sebag, Sitbon, Slama, Taïeb, Tuil, Uzan, Zarka, Zéïtoun et d'autres encore. Tout cela relativise le fait berbère, surtout en milieu urbain, surtout dans l'est du Maghreb et plus encore quand les deux phénomènes se croisent comme ce fut le cas à Tripoli et à Tunis.

\section{Géographie des populations berbérophones}

Vers 1900, au Maroc, à la veille de l'instauration du protectorat français, sur une bande de terre montagnarde de plus de $200 \mathrm{~km}$ de long, d'ouest en est, et de $60 \mathrm{~km}$ de large, du nord au sud, comprise entre l'est de Marrakech, le sud de Demnat et le nord d'Ouarzazate, vivaient 8000 Juifs qui utilisaient les parlers berbères tachelhit (chleuh) à l'ouest et tamazight à l'est, non seulement dans leurs rapports avec l'environnement musulman, mais au sein même de leur univers communautaire et domestique (S. Levy, 1990). Preuve supplémentaire de leur berbérisation totale et ancienne, la découverte récente par le professeur Haïm Zafrani d'une haggada (rituel de la Pâque juive) rédigée en tamazight et, qui plus est, en une langue visiblement archaïque (H. Zafrani, 1988).

Au-delà de cette zone berbérophone, toujours au Maroc, dans l'Anti-Atlas, le Haut Atlas, le Draa', le Sous, vivaient des Juifs, plus nombreux encore, utilisant le berbère majoritairement les parlers tachelhit plutôt que le tamazight - dans leurs contacts avec leurs voisins musulmans, et l'arabe chez eux. Encore qu'à notre sens, ces parlers berbères n'étaient sans doute pas totalement écartés du logis et des rapports intracommunautaires. En tout état de cause, les parlers arabes de ces groupes étaient parfois mal compris des Juifs strictement arabophones car largement pénétrés de mots, d'expressions et de phrases berbères.

Hors du Maroc, on trouvait des Juifs en milieu berbérophone, en Kabylie, au Mzab (parlers zénètes ou zenatiyya), dans le djebel Nefûssa au sud de Tripoli (parlers zénètes). 
17 Les zénètes nomades cavaliers puis chameliers firent leur apparition dans les premiers siècles de l'ère chrétienne dans l'espace correspondant à la Tunisie et l'Algérie, venant de l'est (l'actuelle Libye). Très étirés dans l'espace, de la Cyrénaïque au Maroc oriental, ils pratiquaient des parlers relativement hétérogènes mal compris par les locuteurs de tachelhit et du tamazight.

Ils semblaient n'utiliser le berbère que pour les rapports extra-communautaires, tout au moins au début du XIX ${ }^{e}$ siècle, mais la situation avait pu être différente à une époque plus reculée, et le berbère être la langue domestique, tout au moins en Kabylie et dans le Nefûssa, car, dans le Mzab, les musulmans, généralement bilingues (arabe et berbère), utilisaient volontiers la première langue dans leurs rapports avec les juifs. En Tunisie enfin, la situation semblait au XIXe siècle se rapprocher de celle du Djebel Nefûssa voisin, les juifs se concentrant dans la région restée berbérophone jusqu'au XVIII ${ }^{e}$ siècle, autour de la bourgade de Matmata dans le djebel du même nom.

Dans l'île de Djerba, toujours en Tunisie, les communautés juives des deux bourgades de Ḥâra kbîra et Ḥâra sghîra, aussi longtemps qu'on remonte dans le passé, ne se souviennent pas d'avoir parlé d'autres langues que l'arabe. Pourtant, le sud et l'est de l'île sont restés berbérophones jusqu'à aujourd'hui.

Dans un ordre d'idée voisin, notons que les populations juives de l'arc montagneux du sud-est du Maghreb (Nefûssa, Matmata, djebel Demer) comme celles de Djerba et du Mzab, vivaient, jusqu'au $\mathrm{xx}^{\mathrm{e}}$ siècle, au milieu d'un peuplement musulman non seulement majoritairement berbérophone mais aussi en grande partie îbadîte. Cet environnement spécifique explique, peut-être, certains traits culturels, propres aux Juifs de ces contrées, comme l'extraordinaire sévérité entourant les contacts avec les femmes dans les périodes d'impureté, l'austérité de la vie familiale, l'absence de prostitution, contrairement aux grandes villes, les réticences à l'égard de la musique, l'hostilité marquée à l'égard du chant et de la danse, malgré l'existence de musiciens juifs, une égalité d'humeur enfin et un contrôle de soi en contraste avec les excès verbaux et le débraillé du petit peuple juif de Tripoli, Tunis, Alger, Fès etc.

\section{La répartition géographique des souches berbères}

Les deux listes suivantes permettent une approche géographique de la fréquence des souches berbères au sein de la population juive.

Remarques

Les initiales suivant les souches indiquent les pays ou les régions où elles sont portées: M pour le Maroc, $T$ pour la Tunisie, L pour la Libye, A pour l'Algérois, $C$ pour le Constantinois, $O$ pour l'Oranie et $S$ pour le Sud algérien. Les entités étatiques, Maroc, Libye, Tunisie, paraissent pertinentes également en matière culturelle, d'où notre choix. L'Algérie, en revanche, très étirée en latitude, a été subdivisée en plusieurs sous-ensembles, historiquement et culturellement typés.

24 Souches berbères de manière quasi certaine

Aflalo (M. O). Aknine, Ouaknine, (A, C, O, M.). Amaoua (A, C). Amelard (M). Amgar (M). Amlal, Mlili, Melloul (M, T). Amselem (O, M, A). Amsallag (M, O). Ananou (A). Ankri, Lancry (T, M, O, A). Aoudai (A, O, M). Aouizerate (A, C). Assouline (M). Aurray (M). Azencot (A, S, M). Azeroual (O, S). Azoulay (A, C, O, S, M, T, L). Balouka (A, T). Bellity, Bellilti (M, T). Benhamiyin (M). Bennidder (M). Benittah (M). Bihi (M). Dadi (T). Datchi, 
Ditchi (C). Debda (M). Didi (T). Dodo (M, A). El Fezouati (M). Ergas (T). Fitoussi (A, C, O, T). Friat, Afriat (M, O, A). Gallula (T). Gandus (T). Ghamrasni (T). Guedj, Guez (C, A, O, T). Guest (C). Guetta (T, L, A, C). Hansis (M). Himy (M). Hurighlan (M). Ichi (C). Iddan, Haddan (M). Iddoukh, Boudoukh, Doukhai (M, C). Ifenzi (M). Iferghan, Afrigan (M, T). Iflah (M, C). Ima (A). Innou (M). Issan (M). Issini (M). Isso (M). Ito (C). Izerzel (M). Izri (C, O, M). Jarmon (L, T). Knafo (M). Lolo, Ben Lolo (M, A, T). Loutati (M). Lugassi (M, A, C). Méchiche $(A, C)$. Megaïdès $(T)$. Memmi $(C, T)$. Moumou $(M)$. Nezri, Nezra (M). Ouizgan (M). Ouizman (M, O). Oukrat (A, M, O). Outgorgoch (M). Outmezguit, Outmezguin (M). Sfedj, Sfez (C, T). Sicsou (M, O, C, A, T). Tamsot, Tameshut (M). Taourel (M). Tata (A, C, O, M). Tedgui (O, M, A). Timsit, Temstet, Timestit (A, O, M, T). Toubou (M). Zemour (A, C, T). Zenati (A, O, M). Zerath, Zard, Zert (A, C, O, M, T). Znagui (M). Ziri (M).

25 Souches berbères probables ou partielles

Abisdris (M, O). Acco (M). Agon (S, O, A). Aïnous, Benaïnous (C, T). Akali (C). Amouch (M, O). Amozegh, Benamozegh (M). Amsili (A, M, O). Amstat (M). Aouate (A, C). Archi, Arki $(C, T)$. Atlan $(A, T, C)$. Baranès $(T, L)$. Bedjai $(A)$. Benchetrit $(M)$. Bohbot $(S, M, L)$. Bouahnich, Ahnich, ouahnich (A, M). Bouza-glou (M). Bedjai (A). Benchetrit (M). Dada (M). Dadiah (M). Dadouch (A, C). Demri (T). Ed-Dadsi (M). Ed-Debdubi (M). El Anfaoui $(M)$. El Hyayni (M). Erwiwer (M). Es-Skuri (M). Gaguèch, Ben Gaguèch $(T)$. Ghenassia (O, A, C). Guigui, Benguigui (M, O, A). Haouzi, Haoudji (T, C). Harfi (A, M). Illouz, Beni-louz, Benillouche (M, T). Isgui (M). Isti (O). Isvy (M). Kiki, Kakou, Kakon, peut-être Cacoub (M, T). Lellouche (A, C, T). Lebrati (M, T, O, A). Loufrani, Oufrani (M). Madar (T). Mesguini (C, T). Mesguich (A, C, O). Moha (A, O, M). Naniche (O). Neddour (A, C). Nounou (A, C, O, T). Ohana, Ouanounou (M). Ohayon (M). Ou hamou (C, A, O, M). Ousday (M). Qadra, Ben Qadra (M). Sahij (M). Sisso (M). Sitruk, Strouchi (C, T). Stégassi (M). Stora (M, A). Tamazarti (T). Timgui (M). Tinzi (M). Youyou (A). Zagouri (M). Zozo (M). Zuzuth (M).

On constate, sans véritable surprise, le poids considérable de l'ouest du Maghreb et plus précisément du Maroc, qui paraît bien être la terre d'élection des influences berbères qu'elles soient linguistiques, culturelles ou onomastiques avec, par exemple, dans le cas précis des souches patronymiques, environ 109 souches berbères dans ce dernier pays, sur un total de 150 pour tout le Maghreb soit plus des deux tiers de ce total.

\section{Des mouvements migratoires mal connus}

Ces flux migratoires ne nous intéressent que dans l'exacte mesure où ils peuvent éclairer des problèmes d'onomastique. Dans les zones berbérophones, les juifs paraissent souvent porter plutôt des patronymes arabes. C'est certain au Mzab où ils seraient venus, du XII ${ }^{\mathrm{e}}$ au $\mathrm{XV}^{\mathrm{e}}$ siècle, de zones arabophones, un peu moins net dans le Nefûssa et dans les montagnes des Matmata en Tunisie où paraissent exister quelques patronymes berbères comme Guetta, Gaguech ou Ben Gaguech. Dans le Sud tunisien, on retrouve le patronyme de Demri qui se rapporte au djebel Demer dans la partie méridionale des Matmata, celui de Tamazarti (de Tamezred toujours dans les Matmata), celui de Sroussi (existant aussi en Libye) se rapportant à la région de l'Oued Chrûs dans le Nefûssa.

Quant à la Kabylie, les juifs de cette contrée l'ayant, en grande partie, quittée dès les premiers temps de la colonisation, il est difficile de se faire une idée de leurs patronymes. Il semble bien toutefois que les Memmi, Guedj ou Guez, Didi et peut-être Lellouche fassent référence à cette région. À une époque reculée donc, les juifs y 
auraient été de langue et de culture berbères et peut-être l'étaient-ils restés jusqu'en 1830. Au Maroc, selon certaines traditions, les arabophones des Atlas le seraient seulement devenus, il y a quelques siècles, à la suite d'une immigration continue de familles parlant arabe, langue de prestige. Ces familles, pour partie, seraient venues d'Ifrane, îlot arabophone en plein pays berbère, au sud-ouest de l'Anti-Atlas. Cette tradition parait se confirmer par l'onomastique de la communauté juive du centre. Sur 22 patronymes, seuls 3, Tedghi, Ouhayoun et Iferghan sont en effet de souche berbère (V. Monteil, 1948). À l'inverse, la relative abondance des patronymes berbères dans tout le Maroc s'expliquerait-elle par des mouvements migratoires anciens, mais suractivés aux XIXe et $\mathrm{XX}^{\mathrm{e}}$ siècles, depuis les zones berbérophones rurales et montagnardes vers les villes et particulièrement les villes côtières?

\section{Signification des souches}

En la matière subsistent de vastes zones d'ombre et ce qui suit tient davantage de l'hypothèse et la présomption que de la certitude, sauf cas d'espèce.

On y trouve d'abord des prénoms. Certains comme Itto n'ont rien de juif et sont typiquement berbères; d'autres, en revanche, sont une adaptation berbère de prénoms hébraïques : Bihi pour Abraham, Iddoukh pour Mordekhaï.

On rencontre ensuite une foule de toponymes devenus patronymes, comme Fitoussi, Gallula, Mégaïdès, Tubiana, Sfez ou Sfedj, qui feraient peut-être référence à des villages ou lieux-dits berbères du djebel Nefûssa. Appartiendraient aussi à cette catégorie Azoulay, lieu-dit berbère en Oranie, Tedghi au nord de Tafilalet, Tata village fortifié près d'Agadir, avec aussi une agglomération de ce nom au sud de l'Anti-Atlas.

Les noms de métier sont plus rares. Mentionnons cependant Amsallag, le fabricant de collier (azlag/tazlagt, «collier» en berbère). Les caractéristiques physiques ou autres, les noms de couleur, d'animaux ou de fruits sont plus fréquents.

Gandus signifie veau en kabyle, Azencot a le sens de gazelle dans plusieurs parlers de l'Ouest. Izerzel est son synonyme dans le djebel Nefûssa. Amlal, Melloul, Mlili se réfèrent à couleur blanche, Azeroual à la couleur bleue, avec peut-être le sens de l'homme aux yeux bleus. Azemour, ou Zemmour veut dire olive. Tgorgoch désigne le noyer. Iferghan est le pluriel d'afrag (enclos).

D'autres patronymes sont vraisemblablement berbères, sans qu'il soit possible de déterminer, avec exactitude, leur sens. Tel est le cas de Guedj ou Guez qui s'accrocherait à une racine kabyle ayant le sens de déménager (gaj), de Lellouche, alelluš, « objet brillant de jeu de valeur, toc », en kabyle. Cette interprétation est plus plausible que celle qui veut voir dans ce patronyme une déformation du terme arabe 'alluš (agneau). Il est, en effet, fort improbable qu'une consonne, aussi puissamment articulée que le 'aïn arabe au début de mot, ait pu choir.

Que conclure à l'issu de ce parcours? Il est d'abord certain qu'une onomastique berbère existe chez les juifs du Maghreb ; nullement hégémonique, elle n'est cependant en rien marginale. Ce fait, par lui-même, ne suffit cependant pas à confirmer l'idée d'une origine berbère d'une fraction des Juifs maghrébins, tout en renforçant cette hypothèse.

36 La répartition géographique de notre population, en pays berbère, épouse la nature montagnarde des reliefs avec cependant des zones vides ou presque vides de Juifs 
comme le Rif, le Moyen Atlas marocain, les petits massifs du nord-ouest de l'Algérie, les Aurès. Pour quelles raisons? Les zones de plaine ou de plateaux, jadis domaine des Zénètes nomades venus de l'est, sont également vides. Très grossièrement, les juifs se regroupent surtout dans les aires linguistiques du tamazight et du tachelhit au sein de populations sédentaires ou quasi sédentaires, les Brânés d'Ibn Khaldoun.

Notons enfin la place particulière des Atlas marocains corrélée à l'existence, jusqu'à une date récente (1950), d'un bloc berbérophone montagnard, bloc jadis beaucoup plus étoffé démographiquement avant la descente massive vers les cités, en particulier les cités côtières, aux $\mathrm{XIX}^{\mathrm{e}} \mathrm{et} \mathrm{xx}^{\mathrm{e}}$ siècles. Cete présence rurale, avec même des groupes compacts d'agriculteurs au centre du Haut Atlas est, à première vue, énigmatique et relance l'idée de conversions anciennes au judaïsme.

\section{BIBLIOGRAPHIE}

BRUNSCHVIG R., La Berbérie orientale sous les Hafsides, Paris, Librairie d'Amérique et d'Orient, édition Maisonneuve, 1940, tome I pp. 396-420.

DESPOIS J., Le djebel Nefûssa (Tripolitaire), étude géographique, Paris, Larose éditeur, 1935, p. 137 et sq. EISENBETH M., Les Juifs de l'Afrique du Nord : démographie et onomastique, Alger, imprimerie Carbonnel, 1936.

HANNOUN A., « Historiographie et légende du Maghreb. La Kahena ou la production d'une mémoire ", Annales, Histoire, sciences sociales, 54e année, nº 3, mai-juin 1999.

HIRSCHBERG H. Z., A History of the Jews in north Africa, volume I, From Antiquity to the sixteenth century, Leiden, E.-J. Brill, 1974, p. 145 et sq.

HIRSCHBERG H. Z., « The problem of the judaized berbers », Journal of African history, volume IV, 1963, n 3, pp. 313-339.

LARÉDO A., Les noms des Juifs du Maroc - Essai d'onomastique judéo-marocaine, Madrid, Consejo Superior de Investigaciones Científicos, Instituto B. Arias Montano, 1978.

TOLÉDANO J., La saga des familles. Les Juifs du Maroc et leurs noms, Tel Aviv, éditions Stavit, 1983 ; id. Une histoire de familles. Les noms de famille des Juifs d'Afrique du Nord, Jérusalem, éditions Ramtol, 1998.

LÉVY S., Parlers arabes des Juifs du Maroc : particularités et emprunts. Histoire socio-linguistique et géographie dialectale. Thèse de doctorat d'État en sciences humaines, directeur de recherches Haïm Zafrani, Paris, Université de Paris VIII, 1990, volume I, p. 226 et sq, volume V, p. 1120 et sq. MONTEIL V., « Les Juifs d'Ifrane (Anti-Atlas marocain) ». Hesperis, $1^{\mathrm{er}}$ et $2^{\mathrm{e}}$ trimestres 1948 , tome XXXV, pp. 151-160.

TAÏEB J., « Les Juifs du Maghreb au xix ${ }^{\mathrm{e}}$ siècle à travers leurs patronymes », Actes du V $V^{e}$ congrès international de généalogie juive, à Paris, 13-17 juillet 1997. Paris, cercle de généalogie juive, 1998, pp. 97-107. 
TAÏЕB J., « L'onomastique des Juifs du Maghreb et le fait berbère », Etsi, revue de généalogie et d'histoire séfarades, automne 1998, volume I, n² 2, pp. 3-7.

TALBI M., « Un nouveau fragment de l'histoire de l'Occident musulman, 62-196/682-812, l'épopée d'Al Kahéna ", Cahiers de Tunisie, $1^{\mathrm{er}}$ et $2^{\mathrm{e}}$ trimestres 1971, tome XIX, $\mathrm{n}^{\circ} 73-74$, pp. 19-52.

ZAFRANI H., «Conscience historique et univers judéo-berbère au Maroc » in a Semitic/Afrasian gathering in Remembrance of Albert Ehrman sous la direction de Yaël L. Arbeïtman, Amsterdam, Philadelphie, John Benjamin publishing company, 1988, pp. 499-518.

INDEX

Mots-clés : Aurès, Anthroponymie, Djaraoua, Djerba, Nafusa, Draa, Géographie, Ibn Khaldoun, Judeo-berbère, Kahéna, Langue, Moyen-Atlas, Mzab, Nafusa, Zénètes 